\title{
Harmful Chemicals in the Environment: Measurement, Fate, and Remediation
}

\author{
Jian Lu, ${ }^{1}$ Patrick C. Wilson, ${ }^{1}$ Xianghua Wen, ${ }^{2}$ Qiang Jin, ${ }^{3}$ and Jun Wu ${ }^{4}$ \\ ${ }^{1}$ Indian River Research and Education Center, University of Florida, Fort Pierce, FL 34945, USA \\ ${ }^{2}$ School of Environment, Tsinghua University, Beijing 100084, China \\ ${ }^{3}$ School of Environmental Science and Engineering, Shanghai Jiao Tong University, Shanghai 200240, China \\ ${ }^{4}$ Department of Civil and Architectural Engineering, University of Wyoming, Laramie, WY 82071, USA
}

Correspondence should be addressed to Jian Lu; lujian.leonard@gmail.com

Received 11 May 2015; Accepted 11 May 2015

Copyright (C) 2015 Jian Lu et al. This is an open access article distributed under the Creative Commons Attribution License, which permits unrestricted use, distribution, and reproduction in any medium, provided the original work is properly cited.

Many environmental pollutants that are originally designed for beneficial usage may be harmful to environmental resources and human health by contaminating water, air, and soil. Thus, our special issue aimed at addressing novel analytical methods for monitoring harmful chemicals in the environments as well as various pollution control processes and technologies. We selected the following research articles from the submitted manuscripts to illustrate the current research hotspots in measurement, fate, and remediation of harmful chemicals in environment.

Heavy metals in the environment were investigated by the scientists. R. Mejía-Cuero et al. collected mosses and soil samples from the Metropolitan Area of the Valley of Toluca (MAVT), Mexico, to determine the contents of As, $\mathrm{Cr}, \mathrm{Hg}$, and $\mathrm{Se}$ in the samples. Principal components and cluster analysis methods were employed to reveal the good correlation between metal content in mosses and pollution level in the sampling areas. Y. Lu et al. analyzed the heavy metals including $\mathrm{Cr}, \mathrm{Pb}, \mathrm{Ni}$, and $\mathrm{Zn}$ in soil and plant samples collected from Tongliao, China. The heavy metals in the wastewater-irrigated areas showed higher concentrations than those in the topsoil from the groundwater-irrigated area.

Antibiotics and pharmaceuticals were extensively studied. R. Su et al. investigated treatment of antibiotic pharmaceutical wastewater (APW) using rotating biological contactors (RBC). The results showed that RBC was effective for treating biorecalcitrant wastewater even at low ambient temperature. Y. Xu et al. investigated oxidation of fluoroquinolone (FQ) antibiotic enrofloxacin (ENR) in water by permanganate $[\mathrm{Mn}(\mathrm{VII})]$. Four main oxidation products were identified, showing negligible antibacterial activity when the structures of the products changed. S. Zhang et al. carried out the experiments to explore the distribution of seven antibiotics and ten antibiotic resistance genes (ARGs) in fresh and dried pig feces, biogas slurry, and grape-planting soils from an ecological farm. Positive correlations existed between certain ARGs and the corresponding antibiotics.

Nitrogen pollutants were also paid attention to. G. Shao et al. investigated the effect of controlled irrigation and drainage (CID) at different growth stages of rice on nitrogen (N) contents in rice paddy. The results showed that fertilization could significantly increase the concentration of $\mathrm{NH}_{4}{ }^{+}-\mathrm{N}$ and $\mathrm{TN}$ in surface water but had a little influence on $\mathrm{NO}_{3}{ }^{-}-\mathrm{N}$. $\mathrm{X}$. Zhou et al. studied the seasonal and spatial variation of nitrogen in overlying water of Jinshan Lake, Jiangsu Province, China. Nitrogen concentrations showed significant seasonal variation trends and dramatically changed in 18 sampling sites of Jinshan Lake.

Different techniques including advanced oxidation, electrolysis, biotrickling filter, and complexes preparation were used to explore the measurement, fate, and remediation of pollutants in environments. C. R. Wang et al. studied the effects of current density, initial quinoline concentration, supporting electrolyte, and initial $\mathrm{pH}$ on quinoline mineralization using BDD anode. The biodegradability (the ratio between $\mathrm{BOD}_{5}$ and $\mathrm{COD}$ ) was enhanced from initial 0.02 to 0.57 . P. Wang et al. studied the removal of ethanethiol in biotrickling filter by microorganisms fixed on iron oxidebased porous ceramsite (IPC) under different operating parameters conditions. The results showed that sulfate was 
the main metabolic product and the ethanethiol desulfurization fitted the Michaelis-Menten model. P. Wu et al. prepared complexes of montmorillonite (MT) and kaolinite (KL) with dissolved organic matter (DOM) extracted from landfill leachate under controlled conditions. The results illustrated that the role of montmorillonite and kaolinite complexes with DOM in the retention of pyrene in soils was different. G. Zhu et al. investigated the effects of air flux, pressure, and humidity on generation and concentration of $\mathrm{O}_{3}$ in UV-micro $\mathrm{O}_{3}$ reactor. They also studied degradation of microcystins (MCs) in this reactor. The results proved that UV-micro $\mathrm{O}_{3}$ was a suitable advanced treatment method for degrading dissolved MCs.

Researchers also employed various methods including mathematical models and risk analysis to study harmful chemicals in the environment. G. Chen et al. studied the 2chlorophenol oxidation model in supercritical water using Monte Carlo method for sensitivity analysis. They also identified the parameters of model using two improved differential search (DS) algorithms. The results proved the advantages of strong global search ability of the hybrid methods. Y. Huang et al. investigated the concentrations of pollutants in soil samples collected in and around a dumpsite in Heze, Shandong, China. They also evaluated the potential ecological and health risks of these pollutants. The results showed that $\mathrm{Hg}$ posed a considerable or very high risk. S. Yan et al. studied the dynamics of shallow groundwater level and salinity in the coastal plain region of Jiangsu province, China. Results showed that precipitation, evaporation, and river stage affected the groundwater level in our study area.

New advanced monitoring and remediation techniques will be continuously developed at the basis of the existing various methods to obtain better results in the future. We also hope this special issue will have a long-term impact on the measurement, fate, and remediation of harmful chemicals in various environments.

\section{Acknowledgments}

We would like to thank the Editorial Board for publishing our special issue. We express our thanks to the contributors for their valuable research articles submitted to our special issue. Special thanks and gratitude are extended to the reviewers for their critical and timely assessments of each paper.

Jian $\mathrm{Lu}$

Patrick C. Wilson

Xianghua Wen

Qiang Jin

Jun $W u$ 

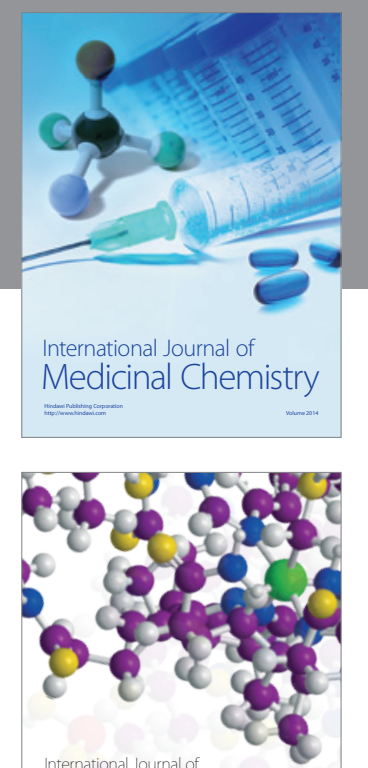

\section{Carbohydrate} Chemistry

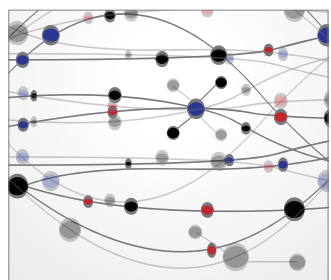

The Scientific World Journal
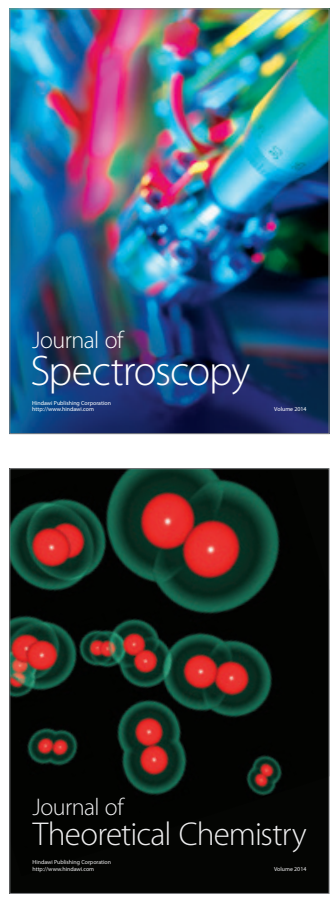
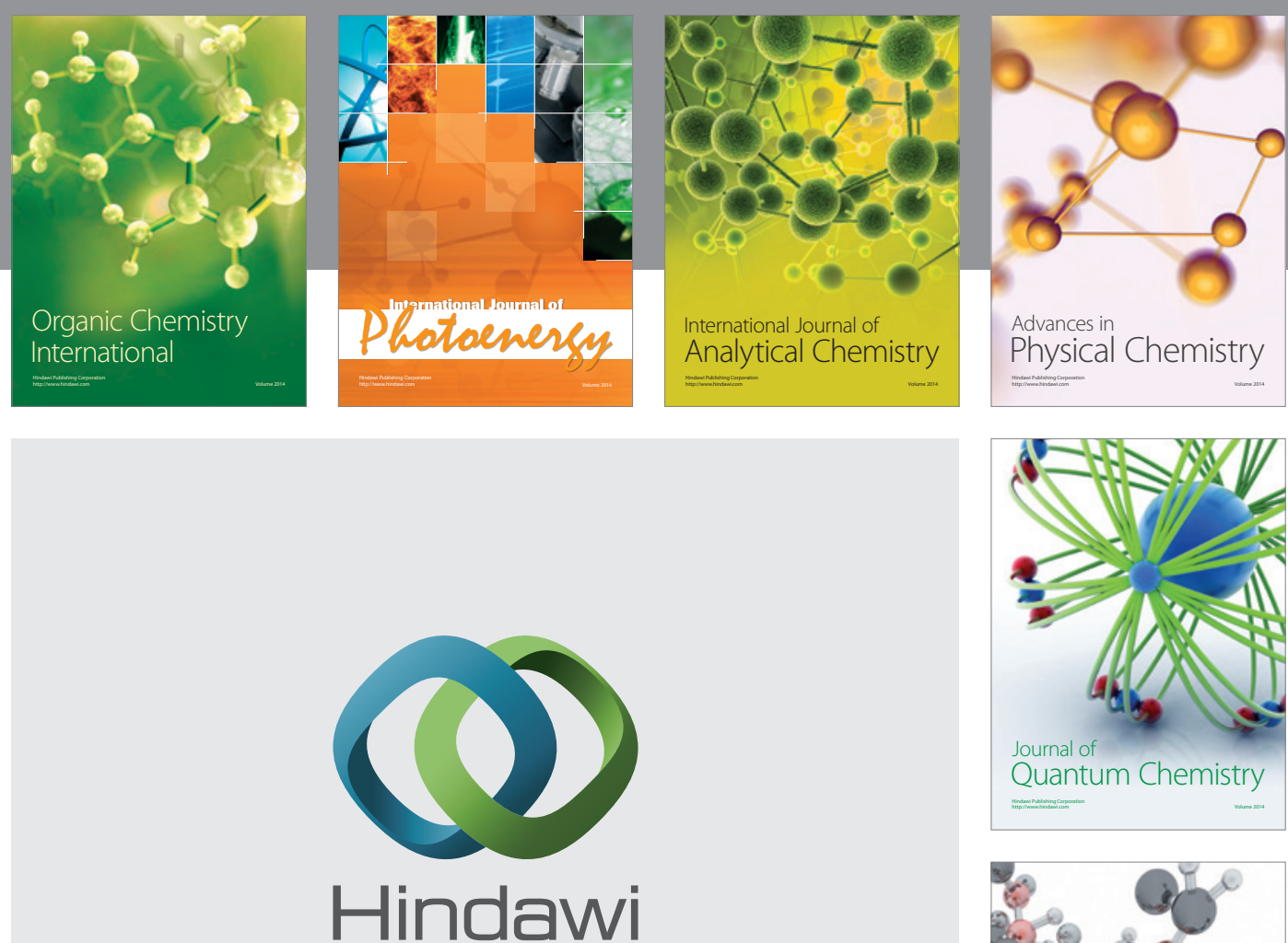

Submit your manuscripts at

http://www.hindawi.com

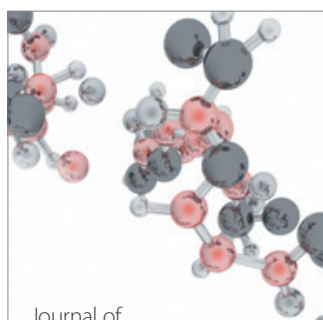

Analytical Methods

in Chemistry

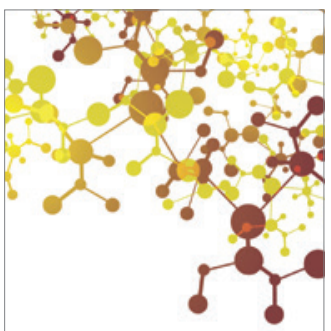

Journal of

Applied Chemistry

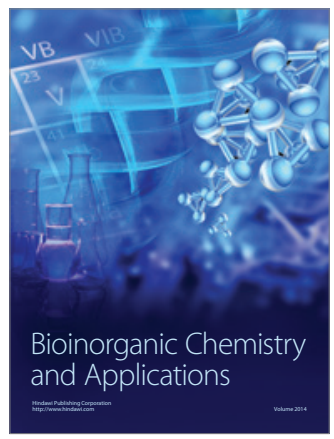

Inorganic Chemistry
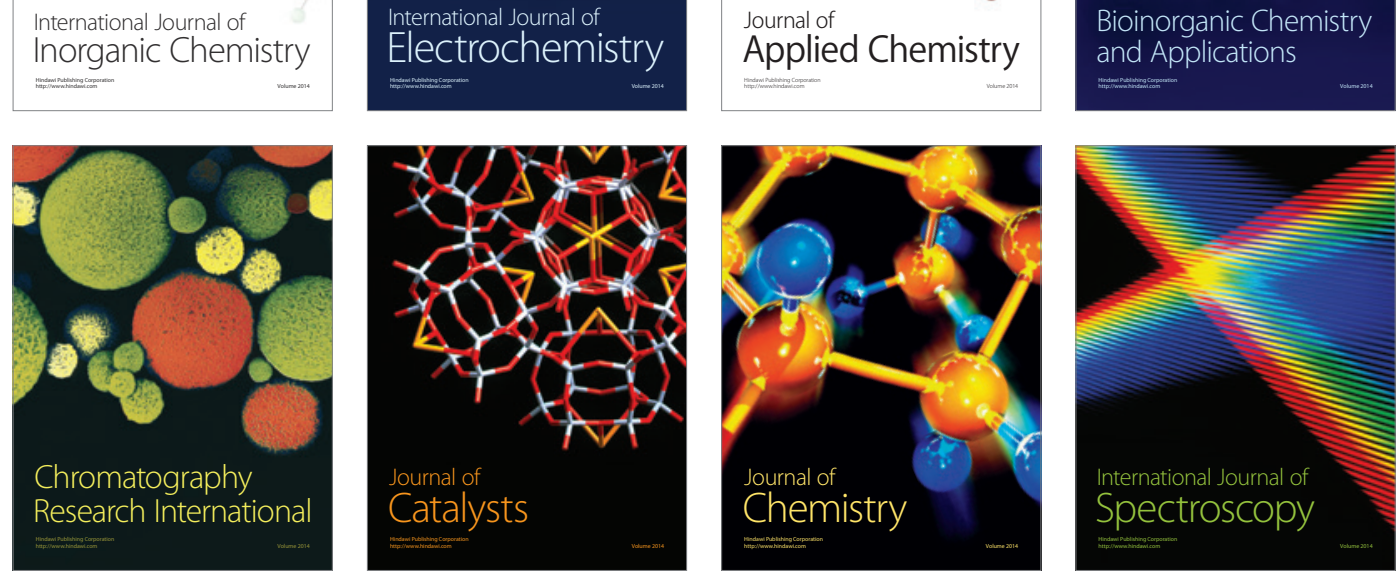